\title{
Absence of compensation and reasoning-like processes in the perception of orientation in depth
}

\author{
WALTER C. GOGEL and JEROME D. TIETZ \\ University of California, Santa Barbara, California
}

\begin{abstract}
When errors are present in the perceived depth between the parts of a physically stationary object, the object appears to rotate as the head is moved laterally (Gogel, 1980). This illusory rotation has been attributed either to compensation (Wallach, 1985, 1987) or to inferential-like processes (Rock, 1983). Alternatively, the perceived distances of and directions to the parts of the object are sufficient to explain the illusory perceived orientations and perceived rotations of the stimulus. This was examined in three experiments. In Experiment 1, a perceived illusory orientation of a stimulus object extended in depth was produced by misleading binocular disparity and was measured at two different lateral positions of the head under two conditions. In the static condition, the head was stationary at different times at each of the two measurement positions of the head. In the dynamic condition, continuous motion of the head occurred between these two positions. In Experiment 2, static and dynamic conditions of illusory stimulus orientation were observed with the head stationary. In Experiment 3, a perspective illusion instead of binocular disparity produced the errors in perceived depth. In no experiment did the perceived orientation of the object differ for the static and dynamic conditions. In the absence of head motion, neither compensatory nor inferential-like processes were available. It is concluded that these processes are not needed to explain either illusory or nonillusory perceptions of the orientation or rotation of stimuli viewed with a laterally moving head.
\end{abstract}

A lateral motion of the observer's head while he or she views a physically stationary, three-dimensional (3-D) object, such as a chair, will result in movement between the parts of the image of the object on the retina. These same changes in the retinal image can be produced either by an appropriate physical rotation of the object, as viewed from a physically stationary head, or by the appropriate simultaneous motions of both the head and the object. How, then, can an observer correctly perceive the physical stationariness or motion of an object physically extended in depth when the information concerning whether the object or the observer is moving is not available in the changing retinal image? One answer is that proprioceptive or efference information regarding the motion or stationariness of the head enables the observer to subtract from the retinal changes that portion of the retinal change attributable to the lateral head motion. The remaining portion, if any, specifies the perceived rotation of the object. This process, sometimes called compensation, or the process of taking into account the observer's own motion,

This paper has been referenced previously in Gogel (1990) as an unpublished manuscript entitled "Limitations of the role of cognitive factors in the perception of orientation in depth." The preparation of this article was supported by Research Grant MH 39457 from the United States National Institute of Mental Health. Correspondence should be addressed to Walter C. Gogel, Department of Psychology, University of California, Santa Barbara, CA 93106-9660. has been applied to a variety of spatial perceptions (Epstein, 1973; 1977; Wallach, 1985, 1987; Wallach \& Flaherty, 1975; Wallach, Stanton, \& Becker, 1974).

The purpose of this paper is to examine the process involved in the perception of the orientation or rotation of an object extended in depth as viewed by a moving or stationary observer, with the observer's motion limited to that of a lateral motion of the head.

If the compensation based on the observer's efference or proprioceptive information with respect to his or her own lateral motion is to result in a correct perception of object rotation, the amount of compensation must differ depending on the physical distance of the object from the observer and its physical extension in depth. This is because the same 3-D object at different distances from the observer, or different objects differently extended in depth at the same distance from the observer, will produce different amounts of retinal change for the same angular rotation of the object or for the same lateral motion of the head. It follows that the accurate perception of a physically stationary or physically rotating object extended in depth, as the head is moved laterally, requires that the perception of the egocentric distances of the parts of the object be correctly perceived. Such an accurate perception of a constant or changing spatial orientation of an object extended in depth could be the result of the observer associating the perceived motion of the head with the retinal changes produced by different perceived egocentric distances of the parts of the object observed 
under conditions in which perceived distance is essentially accurate.

There are instances, however, in which the perceived depth within, for example, a physically stationary object extended in depth and viewed with a laterally moving head is not the same as its physical distance or depth. When this happens, it is the perceived, not the physical, distances between the parts of the object that are responsible for the illusory rotation that is perceived in such an object. This is evident from moving the head laterally during the viewing of physically stationary displays extended in depth such as a 3-D Necker cube, a depth-inverted face mask, or a stereogram in which the physical and perceived depth within the displays differ (Gogel, 1979, 1990; Peterson \& Shyi, 1988; Rock, 1983). One explanation of the illusory perceived rotation concerns anticipation as a consequence of past experience or perhaps as a result of a builtin algorithm. It is supposed that the depth or distance perceived in the physically stationary stimulus extended in depth generates an association or expectation with regard to the direction and amount of retinal change as the head is moved laterally. However, in the case of an error in the perceived distances of the parts of the stimulus, the retinal changes (which are determined by the physical distances of the parts of the stimulus from the observer) will be inconsistent with the associations or expectations. The discrepancy between the anticipated and obtained retinal motions will cause the stimulus to be perceived as rotating. As stated by Wallach $(1987$, p. 5),

It is often noticed that the scene in a large painting appears to rotate as we pass it, or that the head of a portrait seems to turn as if to keep looking at the passing viewer, but this happens only if the painting renders perceived depth realistically. The operation of the compensation process in connection with passing the painting explains this observation.

Rock (1983, p. 7) illustrates a reasoning-like explanation of illusory rotation by the example of a flat, stationary stereogram that appears 3-D and appears to rotate as the head is moved laterally. The perceived rotation is considered to occur in order for the combination of the perceived depth and the lack of retinal change associated with the head motion to be logically consistent. In inferential terms, it is as though the observer solves the problem of the difference between the expected and actual retinal changes by assuming that the stimulus must have changed its motion or orientation as the head was moved laterally. In other words, according to a compensation or reasoninglike explanation, compensation or inferential processes are needed to explain why the errors in the perceived distances of the parts of the stimulus object extended in depth result in an illusory perceived motion of the stimulus as the head is moved laterally. However, it may be unnecessary to describe the processes involved in the perception of stimulus rotation (either illusory or veridical) associated with lateral head motion with respect to compensation, expectation, or perceptual intelligence. An explanation requiring none of these is provided by the theory of phenomenal geometry (Gogel, 1990), which asserts that only three factors are basic to an explanation of spatial phenomena, including the phenomenon of the perception of object rotation as viewed with a laterally moving head. One factor is the observer's perception of the lateral motion of his or her head. A second factor is the change in the perceived direction between the parts of the stimulus object as the head is moved. The third is the perception of the distances of the various parts of the object from the observer. It is asserted that by means of these three factors, the perceived rotation of the stimulus object, whether accurate or in error, is determined.

An example of the application of the theory to an illusory rotation of a stimulus configuration extended in depth and viewed with a lateral motion of the head is shown in Figure 1a. The physical positions of the parts (points) of a physically stationary stimulus in Figure 1a and throughout this study are represented by the solid circles and unprimed notation $e$ and $f$. The perceived positions of the points are shown by the open circles and primed notations $e^{\prime}$ and $f^{\prime}$. The head is shown as physically moving left and right repetitively between Positions 1 and 2 through a physical distance $K$. Throughout this motion of the head, the physically closer point, $e$, is perceived to be at a constant distance, $D_{\mathrm{e}}^{\prime}$, which is greater than its physical distance, $D_{\mathrm{e}}$. The physically more distant point, $f$, is perceived to be at a constant distance, $D_{\mathrm{f}}^{\prime}$, which is less than its physical distance, $D_{\mathrm{f}}$. The physical direction from the head to a stimulus point is indicated by the solid lines. The difference in the physical direction between the two points is $\phi_{1}$ at Position 1 and $\phi_{2}$ at Position 2 of the head. The dashed lines $e_{1}^{\prime} f_{1}^{\prime}$ and $e_{2}^{\prime} f_{2}^{\prime}$ define the limits (terminal positions) of the perceived rotation of the stimulus associated with the head motion, and the perceived angle between these limits is termed $\beta^{\prime}$.

The magnitude of $\beta^{\prime}$ in Figure la can be influenced by the observer's perception $\left(K^{\prime}\right)$ of his or her head motion, the perceived difference in direction between the stimulus points $\phi_{1}^{\prime}$ and $\phi_{2}^{\prime}$, as viewed from the terminal Positions 1 and 2 of the head, and by the perceived distances, $D_{\mathrm{e}}^{\prime}$ and $D_{\mathrm{f}}^{\prime}$, of the points. Figure $1 \mathrm{a}$ is meant to represent the case in which only the perceived distances to points $e$ and $f$ are in error. In other words, it is assumed that $K^{\prime}=K, \phi_{1}^{\prime}=\phi_{1}$, and $\phi_{2}^{\prime}=\phi_{2}$. Figure 1a schematically represents the situation of Experiment 1 of the present study, with point $f$ lower in the visual field than point $e$.

For the purpose of investigating whether compensation or inferential processes can be used to explain the perceived rotation in depth of a stationary stimulus as viewed with a laterally moving head, two kinds of conditions were used in Experiment 1. In one condition, the dynamic, the task of the observer on different trials was to indicate the perceived depth orientation, $e^{\prime} f^{\prime}$, of $e f$ at the instant the head reached Position 1 or Position 2. This was accomplished during the time that the head was moving continuously and repetitively left and right through the distance $K$ while the stimulus points $e$ and $f$ were viewed continuously. In the other condition, the static, the observer on different trials indicated the perceived depth orientation of $e f$ with the head physically stationary at Po- 


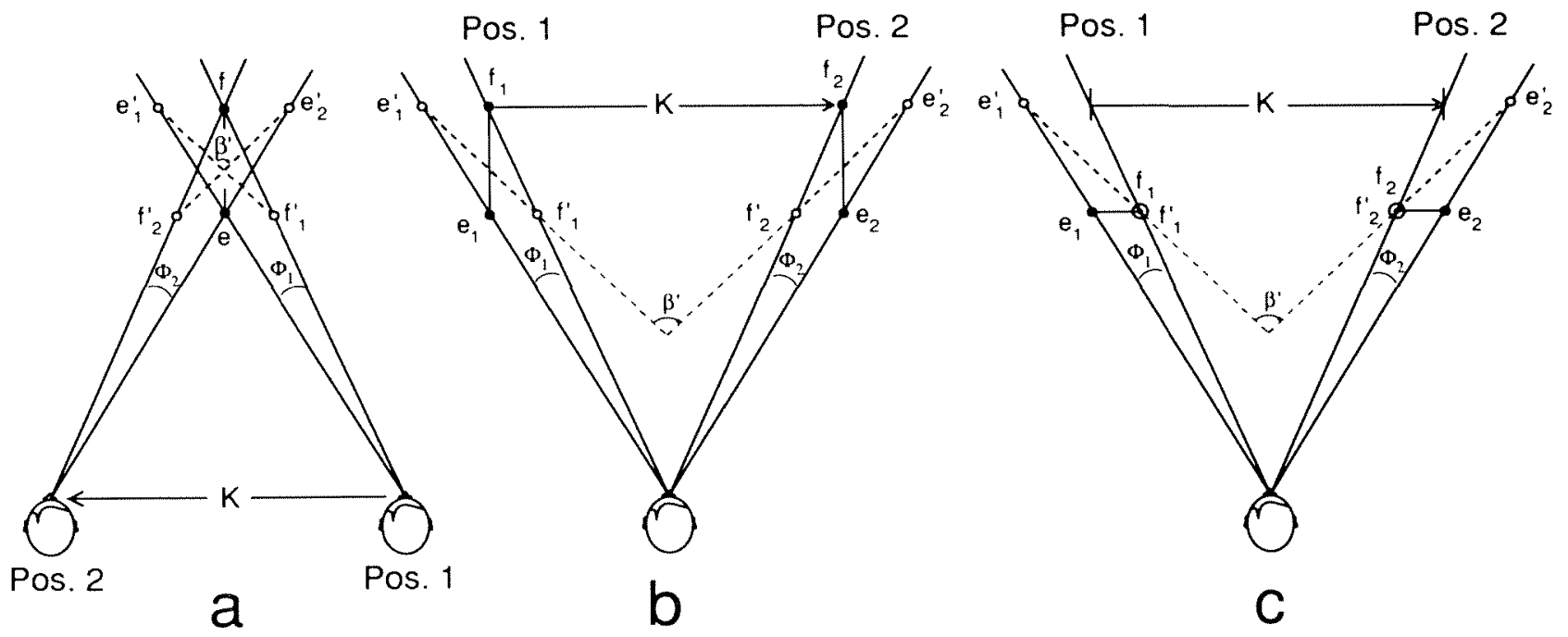

Figure 1. Top-view drawings illustrating the same illusory orientations $\left(e^{\prime} f^{\prime}\right)$ and illusory rotations $\left(\beta^{\prime}\right)$ of a stimulus object $(e f)$ in three different situations. In " $a$," the stimulus object is physically stationary and is viewed with a head laterally moving between Positions 1 and 2 . In " $b$ " and "c," the stimulus object physically moves laterally, and the head is stationary. The situations illustrated in " $a$ " and "c" are used in Experiments 1 and 2, respectively.

sition 1 or Position 2 and without the opportunity to view the stimulus points between these two positions. In this condition, as in the dynamic condition, the difference in the perceived orientation between the two stimulus points at the two static positions of the head will be called $\beta^{\prime}$, even though on any static trial the physically stationary points were seen from only one of the two stationary head positions.

If the perceived orientations of the stimulus points are the same under the static and dynamic conditions, it can be concluded that neither compensation nor inferential processes associated with the lateral motion of the head are needed to explain the perceived illusory rotation of the stimulus. Such a result, however, is not inconsistent with the theory of phenomenal geometry, in which the explanation for the perceived rotation in depth, whether illusory or real, is found in the perceived distances of the stimulus points and their perceived directions from the observer, as provided visually by the available sources of sensory information (cues). The third factor in phenomenal geometry, the perception of the observer's own motion (or stationariness), although involved in many situations, may not be a factor in the perception of orientation or rotation of the stimulus in depth. This is illustrated in the relationship between Figure 1a and Figure 1b. In Figures 1a and $1 \mathrm{~b}$, the stimulus is at the same distance from the observer, and in Figure 1b, the stimulus moves the same lateral extent as does the head in Figure 1a. The information determining the perceived distances and perceived directions of the stimulus points from the observer are the same in the situations of Figures $1 \mathrm{a}$ and $1 \mathrm{~b}$. Thus, according to the theory of phenomenal geometry, the perceived orientation and perceived rotation of the imaginary line connecting the two stimulus points should be the same despite an error in the perceived lateral motion of the head, but only if this error does not produce changes in either $D^{\prime}$ or $\phi^{\prime}$ between the stimulus points. In particular, if the head in Figure 1a, although actually moving through a lateral distance $K$, were perceived to remain stationary, that is, if $K^{\prime}$ were zero, the total perception of the configuration, including that of the perceived lateral motion of the stimulus, would be identical to that in Figure $1 \mathrm{~b}$, but only if the perceived directions and distances of the stimuli are unchanged. Thus, although the common lateral perceived motion or stationariness of the stimulus points can be modified by an illusory perception of the motion or stationariness of the head, the perceived orientation or the change in perceived orientation of the two-point stimulus cannot.

The theory of phenomenal geometry requires that any variable modifying the perceived orientation or rotations of the two-point stimulus illustrated by the situations represented in Figure 1 must produce their effect by modifying the perceptions of distance or direction of the stimulus points. For example, if the distance cue of relative motion parallax were effective in the dynamic conditions of Figure 1a, the errors in the perceived distances of the points, and thus the perceived rotation, would be less in the dynamic relative to the static condition. This is not apt to occur, however, in these situations of Experiments 1 and 2 because the errors in the perceived distances of points $e$ and $f$ were achieved by manipulating the very effective cue of binocular disparity. It is unlikely that a motion parallax involving only two stimulus points would substantially modify a contrary perceived depth supported by binocular disparity. 


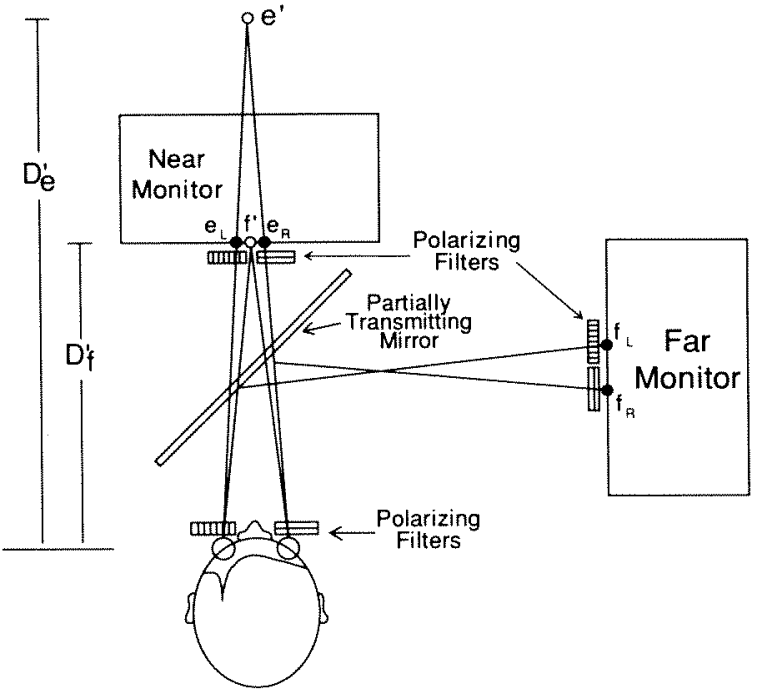

Figure 2. Top-view drawing of the apparatus used in Experiment 1 showing the method of producing the simulated distances of $e^{\prime}$ and $f^{\prime}$ illustrated in Figure 1a. Each simulated point is the result of the observer binocularly fusing a pair of luminous points on either a near or a far monitor. By using polarizing filters on the monitor and in front of the observer's eyes, only one of a pair of points is seen by each eye. The simulated (convergence) distance produced by using the near monitor is more distant than that produced with the far monitor. The partially transmitting mirror aligns the simulated points so that, at the midposition of the head (midway between Positions 1 and 2 of Figure 1a), the simulated points are in the midsagittal plane of the observer.

\section{EXPERIMENT 1}

\section{Method}

\section{Observers}

The observers were 12 graduate students ( 7 men and 5 women) who were paid for their participation. All had at least 20/30 acuity in both eyes, near and far, and at least 22.08 - $\mathrm{sec}$ arc of stereopsis, as measured on a Keystone orthoscope. Several wore contact lenses, none wore glasses, and all were naive with regard to the purpose of the experiment.

\section{Apparatus}

Stimuli. The apparatus used to generate the stimuli consisted of a Commodore 64 computer and two Model VM-173 Hitachi composite video monitors. As is illustrated in Figure 2, the two stimulus points $e$ and $f$ were generated on separate monitors: a near monitor at $1.00 \mathrm{~m}$ from the observer's eyes and a far monitor at $1.43 \mathrm{~m}$ from the observer's eyes. The simulation of a binocularly fused point ( $e^{\prime}$ or $f^{\prime}$; represented by the open circles) in perceived space was generated by two laterally separated luminous points physically located on a display monitor (indicated by a pair of filled circles). Of each pair of points on a monitor, one was visible only to one eye, and the other was visible only to the other eye. This was accomplished by means of polarizing filters located at the monitor screen and also in a frame fixed to the headrest and located $5 \mathrm{~cm}$ in front of the observer's eyes. The binocular fusion of the two points on the near monitor (uncrossed convergence), labeled $e_{\mathrm{L}}$ and $e_{\mathrm{R}}$ in Figure 2, and on the far monitor (crossed convergence), labeled $f_{\mathrm{L}}$ and $f_{\mathrm{R}}$, resulted in the simulated point produced by the near monitor $\left(e^{\prime}\right)$ appearing to be more distant than that produced by the far monitor $\left(f^{\prime}\right)$. To avoid $e^{\prime}$ and $f^{\prime}$ being interposed when the head was at the middle of its motion, the points on the far mon- itor were positioned directionally $2.08^{\circ}$ below the observer's eye level, whereas the points on the near monitor were located $2.43^{\circ}$ above it. The lateral separations of each pair of the points on the monitors were calculated to simulate a convergence distance (based on an interocular distance of $6.4 \mathrm{~cm}$ ) of $1.00 \mathrm{~m}$ for the points on the far monitor and $1.43 \mathrm{~m}$ for the points on the near monitor (a reversal of their physical distances). Also, as shown in Figure 2, a partially reflecting, partially transmitting first surface mirror aligned the two binocularly fused points of light (shown by the small open circles) so that they would be in the sagittal plane of the observer when the observer's head was laterally midway between Positions 1 and 2. The resulting binocular perception was of two white points of light, $e^{\prime}$ and $f^{\prime}$, at different distances, with the perceptually more distant point perceptually higher in the visual field. The brightness of the points, as viewed through the polarizing filters, was $1.15 \mathrm{log}$ units above foveal threshold under the average adaptation conditions of the experiment. Neither the mirror nor any object or surface except the two simulated binocularly viewed points of light were visible to the observer.

The observer's head was placed in a head-chinrest assembly that could be moved left and right on ball-bearing rollers through $20 \mathrm{~cm} \pm$ $1 \mathrm{~mm}$ or could be pinned at one or the other extreme head position. The arrival of the head at the right (Position 1) or left (Position 2) extremes of head motion activated small lights at the experimenter's position (shielded from the observer) to inform the experimenter that the head was being moved laterally through the full amount. The polarizing filters in front of the two eyes were mounted on the frame fixed to the head and chinrest to keep the filters appropriately aligned in the observer's frontoparallel plane despite the small head rotations that might have occurred in order to fixate a stimulus point during the extremes of head motion.

Response-measurement apparatus. The task of the observer was to indicate the perceived orientation of an imaginary straight line connecting the perceived points $e_{1}^{\prime} f_{1}^{\prime}$ and $e_{2}^{\prime} f_{2}^{\prime}$ of the two-point simulated stimulus. To accomplish this, a response device, illustrated in Figure 3, was located $90^{\circ}$ to the right of the observation position, with this device and its surround illuminated by a floor lamp that was turned on following the observation of the stimulus. The response device consisted of both a square plate $(16.7 \mathrm{~cm}$ on a side) whose slant in depth could be rotated around its central, horizontal axis by turning a knob (the slant adjustment) and a thin white metal rod (15.3 cm long) that could be rotated around its midpoint in the

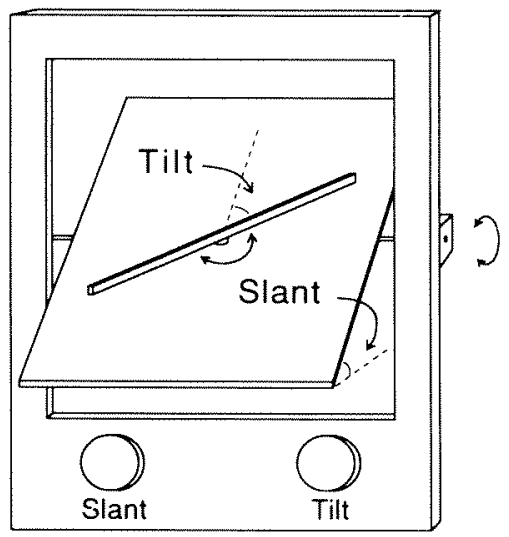

Figure 3. Adjustment apparatus that was used to measure perceived tilt and perceived slant in Experiments 1 and 2. The axis of rotation of the adjustment used to measure perceived tilt is perpendicular to the surface of the plane used to measure perceived slant. The adjustments shown are an approximately $45^{\circ}$ clockwise adjustment measuring a perceived tilt and an approximately $45^{\circ}$ adjustment measuring a perceived slant. 
adjusted plane of the plate by turning a second knob (the tilt adjustment). The slant and tilt axes were each connected to separate rotary potentiometers that were read by the computer's $A / D$ inputs and converted into degrees $\left( \pm 1^{\circ}\right)$ of angular slant and tilt. The perception of slant as measured by the adjustment of the plate was a consequence of the stimulus cue of binocular disparity and the difference in height of the two stimulus points. This measure is not the major interest of the present study. The adjusted tilt of the white rod in the plane of the slant plate, however, indicates the perceived orientation $e_{1}^{\prime} f_{1}^{\prime}$ and $e_{2}^{\prime} f_{2}^{\prime}$ of Figure 1-the major variable of the study - with the computed difference in the perceived tilt between $e_{1}^{\prime} f_{1}^{\prime}$ and $e_{2}^{\prime} f_{2}^{\prime}$ to be called the perceived rotation, $\beta^{\prime}$, regardless of whether this perceived difference was obtained from the two head positions under either dynamic or static conditions.

\section{Procedure}

In the observation booth, the observers were shown how to use the apparatus that indicated the magnitude of perceived slant and tilt. There were five trials in each of two sessions, with each trial consisting of one slant and one tilt response. The first trial was a practice trial in which the conditions of the immediately following experimental trial were used. The experiment consisted of three independent variables: head condition (static or dynamic), head position (Position 1 or Position 2) at which the perceived slant or tilt of the imaginary straight line connecting the stimulus points was judged, and response type (slant or tilt). Two trials were run consecutively at the same head position, after which the alternative position was used. The two sessions were separated by approximately 1 week and differed only with respect to the head condition (static or dynamic). Half of the observers received the static condition in the first session followed by the dynamic condition in the second. This order was reversed for the remaining observers. The time between closing the shutter on one trial and opening it again for the next trial was approximately $25 \mathrm{sec}$, depending on how long it took the observer to respond.

Static condition. The chinrest was pinned at the left or right extreme position (Position 1 or Position 2 of Figure 1a), and the viewing aperture was opened to present the two points of light. The observer was asked to note the direction and amount of slant and tilt of the imaginary line connecting the two stimulus points. The viewing aperture was then closed, the observation booth was illuminated, and the observer turned to the right and adjusted the response apparatus to indicate first the perceived slant in depth and then the perceived tilt of the imaginary connecting line. Following this, the observer returned both components of the measurement device to the vertical position and placed his or her head in the chinrest to begin the next trial. After completing two slant and tilt measurements at the same head position (Position 1 or Position 2), two additional response measures of slant and tilt were obtained at the remaining head position (Position 2 or Position 1).

Dynamic condition. The procedure for the dynamic condition was the same as that for the static condition except that the head and chinrest were freed, permitting the observer to move his or her head laterally between the stops at Positions 1 and 2. There was a 2 -sec interval between clicks of a metronome used to guide the arrival of the head at the right and left stop per half cycle of head motion. The observer was instructed to note the perceived slant and tilt of the imaginary line connecting the two stimuli when, on different trials, the head was at the right extreme of head motion (Position 1) or at the left extreme of head motion (Position 2). Half of the observers completed the adjustments of the measuring device twice at Position 1 followed by twice at Position 2. The remaining observers had the reverse order.

\section{Results}

The average results from Experiments 1 and 2 are shown in Table 1. The mean perceived tilts and slants were obtained by using the average of the two repeated measures from each observer. The perceived rotation $\left(\beta^{\prime}\right)$ was computed from the differences in the perceived tilts between Positions 1 and 2 of the head. For the perception of tilt, a counterclockwise result is positive; a clockwise result is negative. For the perception of slant, the perceptually farther point always was perceived as being higher in the visual field than was the perceptually nearer point, with this result shown as positive. The above comments concerning the results apply throughout Table 1 -that is, they apply to both Experiments 1 and 2 . The results exclusively from Experiment 1 are shown in the top half of Table 1. An analysis of variance (ANOVA) was applied separately to the slant and tilt responses for the following variables: head condition (static or dynamic), head position (Position 1 or Position 2), and first or second measures of slant or tilt. For the slant-in-depth responses, none of these factors was statistically significant at the .05 level. This is

Table 1

Results From Experiment 1 and 2 (in Degrees)

Experiment 1

\begin{tabular}{|c|c|c|c|c|c|c|c|c|c|c|c|c|}
\hline \multirow[b]{3}{*}{ Response } & \multicolumn{6}{|c|}{ Stationary (Static) Head } & \multicolumn{6}{|c|}{ Moving (Dynamic) Head } \\
\hline & \multicolumn{2}{|c|}{$\begin{array}{l}\text { Head Right } \\
\text { (Position 1) }\end{array}$} & \multicolumn{2}{|c|}{$\begin{array}{c}\text { Head Left } \\
\text { (Position 2) }\end{array}$} & \multicolumn{2}{|c|}{$\beta^{\prime}$ Static } & \multicolumn{2}{|c|}{$\begin{array}{l}\text { Head Right } \\
\text { (Position 1) }\end{array}$} & \multicolumn{2}{|c|}{$\begin{array}{c}\text { Head Left } \\
\text { (Position 2) }\end{array}$} & \multicolumn{2}{|c|}{$\beta^{\prime}$ Dynamic } \\
\hline & $M$ & $S D$ & $M$ & $S D$ & $M$ & $S D$ & $M$ & $S D$ & $M$ & $S D$ & $M$ & $S D$ \\
\hline $\begin{array}{l}\text { Tilt } \\
\text { Slant }\end{array}$ & $\begin{array}{l}20.36 \\
20.70\end{array}$ & $\begin{array}{l}4.54 \\
7.15\end{array}$ & $\begin{array}{r}-17.04 \\
18.63\end{array}$ & $\begin{array}{l}6.45 \\
7.46\end{array}$ & 37.40 & 8.91 & $\begin{array}{l}20.19 \\
21.81\end{array}$ & $\begin{array}{r}7.02 \\
10.97\end{array}$ & $\begin{array}{r}-17.96 \\
21.35\end{array}$ & $\begin{array}{r}9.72 \\
10.94\end{array}$ & 38.15 & 13.28 \\
\hline
\end{tabular}

Experiment 2

\begin{tabular}{|c|c|c|c|c|c|c|c|c|c|c|c|c|}
\hline \multirow[b]{3}{*}{ Response } & \multicolumn{6}{|c|}{ Stationary (Static) Stimulus } & \multicolumn{6}{|c|}{ Moving (Dynamic) Stimulus } \\
\hline & \multicolumn{2}{|c|}{$\begin{array}{l}\text { Stimulus Left } \\
\text { (Position 1) }\end{array}$} & \multicolumn{2}{|c|}{$\begin{array}{l}\text { Stimulus Right } \\
\text { (Position 2) }\end{array}$} & \multicolumn{2}{|c|}{$\beta^{\prime}$ Static } & \multicolumn{2}{|c|}{$\begin{array}{l}\text { Stimulus Left } \\
\text { (Position 1) }\end{array}$} & \multicolumn{2}{|c|}{$\begin{array}{c}\text { Stimulus Right } \\
\text { (Position 2) }\end{array}$} & \multicolumn{2}{|c|}{$\beta^{\prime}$ Dynamic } \\
\hline & $M$ & $S D$ & $M$ & $S D$ & $M$ & $S D$ & $M$ & $S D$ & $M$ & $S D$ & $M$ & $S D$ \\
\hline $\begin{array}{l}\text { Tilt } \\
\text { Slant }\end{array}$ & $\begin{array}{l}17.27 \\
20.52\end{array}$ & $\begin{array}{l}3.96 \\
6.68\end{array}$ & $\begin{array}{r}-17.04 \\
20.34\end{array}$ & $\begin{array}{l}2.33 \\
7.42\end{array}$ & 34.31 & 5.64 & $\begin{array}{l}18.69 \\
20.10\end{array}$ & $\begin{array}{l}4.28 \\
7.50\end{array}$ & $\begin{array}{r}-17.50 \\
19.78\end{array}$ & $\begin{array}{l}3.37 \\
9.15\end{array}$ & 36.19 & 6.30 \\
\hline
\end{tabular}

Note-According to $t$ tests, all mean values of tilt and slant differed significantly from zero at a probability level of less than .0005 . In Position 1, the perceptually farther point $\left(e_{1}^{\prime}\right)$ always was perceived to be to the left of the perceptually nearer point $\left(f_{i}^{\prime}\right.$; a positive tilt). In Position 2 , the perceptually farther point $\left(e_{2}^{\prime}\right)$ always was perceived to be to the right of the perceptually nearer point ( $f_{2}^{\prime}$; a negative tilt). (See Figures la and $1 \mathrm{c}$.) 
to be expected, since the perception of the slant of the imaginary line connecting the two stimulus points was determined by the constant conditions of binocular disparity and vertical separation between the points. For the tilt responses, only the head position (right or left), that is, Position 1 or Position 2 in Figure 1a, was statistically significant $[F(1,11)=249.36, p<.0005]$, with neither of the other variables, or any interactions, significant at the .05 level.

An ANOVA of the $\beta^{\prime}$ calculations for Experiment 1 (as shown in the upper half of Table 1) resulting from the differences between the right and left tilt judgments was performed by using the variables of motion condition (static or dynamic) and first or second measure. Neither the variables nor their interaction was significant at the .05 level. In particular, the difference between the dynamic and static $\beta^{\prime}$ shown in Table $1\left(38.15^{\circ}-37.40^{\circ}\right)$ resulted in $F(1,11)=.047, p=.831$. This experiment offers no support for the notion that the lateral motion of the head in the dynamic condition, as contrasted with the static condition, modified the perceived tilt or the change in perceived tilt $\left(\beta^{\prime}\right)$ of the imaginary straight line connecting the two stimulus points.

\section{EXPERIMENT 2}

It is possible that the same perceived tilts occurred in the static and dynamic condition of Experiment 1 because the observer was aware in the static conditions that the same stimulus was being viewed from the different positions (Positions 1 or 2) of the head. That is, it is possible that compensation or reasoning-like effects can occur from memories of prior static displacements of the head, as well as from continuous (dynamic) head motions. This could be tested in the situation of Figure $1 \mathrm{~b}$. In this case, the head is kept physically, as well as perceptually, stationary, and the stimulus object either is physically displaced (statically) or is physically continuously moved (dynamically) through a distance $K$ between Positions $e_{1} f_{1}$ and $e_{2} f_{2}$, where $K$, as shown in the drawings of Figure 1 , is always the same. Since the head is always physically stationary in Experiment 2, compensation for head motion is not possible. Thus, if compensation or inferential processes were present in both the static and dynamic conditions of Experiment 1, the perceived tilts and computed rotations from Experiments 1 and 2 should differ.

In attempting to produce the stationary head and moving stimulus condition of Figure $1 \mathrm{~b}$, however, a technical difficulty (noted below) was encountered that limited the conditions used in Experiment 2 to those of Figure 1c rather than Figure 1b. The only difference between Figures $1 \mathrm{c}$ and $\mathrm{lb}$ is that the stimulus points $e$ and $f$ on the monitors are at the same physical distances in Figure $1 \mathrm{c}$, whereas in Figure $1 \mathrm{~b}$, point $f$ is physically more distant than point $e$. Although the accommodative difference between points $e$ and $f$ is greater in Experiment 1 than in Experiment 2, the convergence and binocular disparity between the simulated points was identical in both experiments. It is very likely that the effectiveness of the binocular disparity would remove any significant contribution of accommodative differences to the perceived depth between the stimulus points in either experiment. Thus, it seems that the situation of Figure 1c can substitute for that of Figure $1 \mathrm{~b}$ in a comparison of the results of Experiments 1 and 2.

\section{Method}

\section{Observers}

The observers were the same 12 graduate students used in Experiment 1 . All were uninformed about the purpose of the experiment and were paid for participating.

\section{Apparatus}

Experiment 2 used the same computer, display monitors, and response device used in Experiment 1. Two points of white light, viewed binocularly in a totally dark surround, were presented at simulated (convergence) distances of 100 and $143 \mathrm{~cm}$. The point at the more distant simulated distance (greater perceived distance) was higher in the visual field, as it was in Experiment 1. Because the stereoscopically generated pair of points was required to move laterally on the monitor screens through a substantial distance, to ensure that each point remained behind its polarizing filter, each simulated point was generated as follows. The physical distance of both monitors was $100 \mathrm{~cm}$ from the observer. The lower point at both a physical and a simulated near convergence distance of $100 \mathrm{~cm}$ was generated by a single point of light and was seen with both eyes. The upper point was generated binocularly by means of polarizing filters and was a single point on each of the two monitors. The monitor generating both the binocularly seen lower point and the upper point seen by the right eye was located in front of the observer and was viewed by transmission through the partially transmitting mirror. The monitor generating the upper point seen by the left eye was located in front of and to the right of the observer and was viewed by reflection from the mirror. The monitors were offset horizontally to simulate a convergence distance to the upper point of $143 \mathrm{~cm}$. A neutral density filter was placed over the lower point to equate its brightness to that of the other point, which had polarizing filters in front of it.

Dynamic and static conditions were also used in Experiment 2. In the static condition, the stimulus points were presented either to the right or left of the observer's straight-ahead direction, with the head physically stationary at the center position. The visual direction of each point was calculated to duplicate the directions used in Experiment 1 when the head was at the extreme positions. In the dynamic condition of Experiment 2, the observer's head was also stationary at the center position, but the pair of binocularly viewed stimulus points were made to move repetitively left and right so as to duplicate the changes in visual direction that the observer experienced while moving the head in the dynamic condition of Experiment 1 . The time for the points to travel from one side to the other in Experiment 2 (approximately $2.8 \mathrm{sec}$ ) was greater than the time for the head to move from one side to the other in Experiment 1 . The slower speed in Experiment 2 was necessary for the computer to produce a smooth stimulus motion on the monitor screen. In Experiment 2, as compared with Experiment 1, neither the change in accommodation of point $f$ nor the slower motion of the stimulus points was expected to modify the perceived tilts or the computed changes in perceived tilts $\left(\beta^{\prime}\right)$ obtained in the two experiments.

\section{Procedure}

The procedure and order of conditions were identical to those of Experiment 1, with the exception that the terms "dynamic" and "static" in Experiment 1 refer to the observer's head motion or position, whereas in Experiment 2, it refers to the lateral motion or position of the pair of binocularly viewed stimulus points, the 
observer's head being physically stationary at the center of the stimulus motion or displacement throughout the experiment. As in Experiment 1 , the observer's task was to indicate the perceived slant and perceived tilt (in that order) of an imaginary straight line connecting the lower and upper stimulus points when they were at the extremes of stimulus motion or at the two static positions (Position 1 or Position 2). In the dynamic conditions of Experiment 2, as in Experiment 1, the right or left extreme positions at which the judgments were to be made were specified by the experimenter before each trial. The time between trials was approximately $26 \mathrm{sec}$.

\section{Results}

The mean slant and tilt reports, averaged over the two response measurements of slant or tilt under each condition in Experiment 2, together with the mean computed change in perceived tilt $\left(\beta^{\prime}\right)$, are shown in the lower half of Table 1 .

An ANOVA was applied separately to the tilt and slant results for the following variables: stimulus condition (static or dynamic), stimulus position (Position 1 or Position 2), and first or second measure of slant or tilt. For the slant-in-depth response, none of these factors were statistically significant at the .05 level. This was expected, since the perception of the slant of an imaginary straight line connecting the stimulus points was determined by the constant conditions of binocular disparity and vertical separation between the binocularly viewed points. For the tilt in the plane of the slant, stimulus position (1 or 2 , as shown in Figure 1c) was statistically significant $[F(1,11)$ $=462.81, p<.0005]$, with neither of the remaining variables, or any interactions, significant at the .05 level. An ANOVA of the $\beta^{\prime}$ values (also shown in the lower half of Table 1) resulting from the difference in degrees between left and right tilt judgments was performed by using the variables of stimulus motion condition (static or dynamic) and first or second measures. Neither the variables nor their interaction was statistically significant. In particular, the difference between the dynamic and static $\beta^{\prime}$ shown in the lower half of Table $1\left(36.19^{\circ}-34.31^{\circ}\right)$ resulted in $F(1,11)=3.03, p=.109$. This experiment offers no support for the hypothesis that the dynamic stimulus condition, as contrasted with the static stimulus condition, modified the perceived orientation or change in orientation $\left(\beta^{\prime}\right)$ of an imaginary line connecting the two stimulus points.

\section{Discussion}

Comparisons of results from Experiments 1 and 2. A comparison of the upper and lower halves of Table 1 indicates that the perceptions of slant, tilt, or rotation $\left(\beta^{\prime}\right)$ obtained in Experiments 1 and 2 were very similar. In the top half of Table 1 (Experiment 1), in which the head was moving or was statically displaced and the stimulus was stationary, Positions 1 and 2 refer to the right and left positions of the head, respectively. In the bottom half of Table 1 (Experiment 2), in which the stimulus was moving or was statically displaced and the head was stationary, Positions 1 and 2 refer to the left and right posi- tions of the stimulus, respectively. Thus, as indicated by Figures 1a and 1c, the expected and obtained perceived tilts from Experiments 1 and 2, as listed in the corresponding column of Table 1 , are essentially the same.

An ANOVA was applied to test the significance of differences between the mean values of $\beta^{\prime}$ obtained for the variables (motion, either static or dynamic, and first or second measures of tilt) of Experiments 1 and 2 . None of the values of $\beta^{\prime}$ for any of these variables or their interactions were statistically significant. In Experiment 2, neither head motion nor head displacement was present. A comparison of the results from Experiments 1 and 2 provided no evidence that compensation or reasoning-like processes were important in producing the illusory orientations or rotations of the stimulus in either experiment. Instead, the determining factors were the perceptions of the distance and direction of the parts (points) of the stimulus in the different situations and conditions of Experiments 1 and 2 .

Application of the theory of phenomenal geometry. It will be noted that the difference between the physical and simulated depth between points $e_{1}$ and $f_{1}$ in Experiment 2 (see Figure 1c) was only half that in Experiment 1. Nevertheless, the perceived tilts and rotations from the two experiments did not differ substantially or significantly because the perceived distances (and very likely the perceived directions) of the stimulus points in Experiments 1 and 2 were the same. The basic determiners of the perceived tilts and rotations in both experiments were the perceived distances and perceived directions of the points and not whether, or how much, these perceptions differed from the physical conditions. Errors in the perceived distances or perceived directions of the parts of the stimulus were important in modifying the derived perceptions of tilt and rotation only as they had consequences for the perceived distances or perceived directions of the points. This agrees with the theory of phenomenal geometry, in which perceived distance and direction, not errors in perceived distance or direction, are basic variables that determine the derived perceptions of orientation or rotation. It often is of interest to specify when and how much the perceptual and physical worlds differ. Nevertheless, to understand a derived perception such as tilt or rotation, the explanation must be in terms of the basic perceptual variables, in this case, perceived distance and direction, however these are determined. This does not mean, however, that relationships between basic and derived perceptions are independent of relationships between physiological events. It is expected that every unique perceptual event reflects a unique physiological condition. But an explanation with respect to either physical or simulated events is, from the viewpoint of the theory of phenomenal geometry, incomplete. For example, in Figure 1a, suppose that $e_{1}^{\prime} f_{1}^{\prime}$ and $e_{2}^{\prime} f_{2}^{\prime}$ represented physically accurate (rather than illusory) perceptions of tilts and rotation. If this occurred, the observer's perception of the tilts and rotations would remain as it was represented in the figure, as long as the per- 
ceived distances and directions of the points were the same as those present when the errors in perceived depth were substantial.

\section{EXPERIMENT 3}

The purpose of Experiment 3 was to provide a situation similar to that used with the static and dynamic conditions of Experiment 1, except that the error in the perceived depth orientation (perceived tilt) of the display was the result of relative size (perspective) cues of distance caused by the use of an Ames trapezoidal window (Ames, 1951). Positioning the trapezoidal window with its small end closer to the observer than its large end (as shown in Figure 4) results in a perception (particularly if the observation is monocular) that its perceived orientation in depth (perceived tilt) is reversed from its physical tilt. When this illusory orientation is perceived, moving the head laterally results in the window appearing to rotate in a manner similar to that indicated by the dashed lines in Figure 1a. The same magnitude of change in perceived tilt in depth (a perceived rotation of the window clockwise or counterclockwise, depending on the direction of head movement) from the median (midsagittal) plane between the two extremes of head motion for the static and dynamic head conditions would indicate that an explanation of perceived orientation or rotation with respect to the theory of phenomenal geometry is not limited to the depth cue of binocular disparity.

\section{Method}

\section{Observers}

The observers in the experiment were 12 graduate students who were paid for their participation. Nine of these had participated in Experiments 1 and 2. All were naive regarding the purpose of the experiment and all satisfied the visual acuity requirements of the previous experiments.

\section{Apparatus}

The observer sat on a stool inside a lightproof booth and viewed the stimulus through an occludable aperture. The observations were made with the head held in a chinrest assembly that could be moved laterally through a distance of $35.1 \pm 0.5 \mathrm{~cm}$. In one of two sessions, the observer moved the head repetitively from side to side in time with metronome clicks spaced at 3.0-sec intervals (the dynamic condition). In the other session (the static condition), the chinrest and head were held stationary at either the right or left extreme position (Position 1 or 2 in Figure 4).

The trapezoidal window contained six panes, three in the upper and three in the lower half of the window. The center of the window was at eye level and was viewed with the right eye only, in an otherwise totally dark surround. As is illustrated in Figure 4, the trapezoidal window was physically located to the left and forward of the observer's position, and the image of the window was reflected into the observer's right eye by a partially transmitting, partially reflecting mirror oriented at $45^{\circ}$ to the observer's midsagittal plane at the midpoint of the motion of the head. When the head was at the midpoint of its path of lateral motion, the window appeared to be oriented in a sagittal plane passing through the observer's right eye. The trapezoidal window was constructed from two white electroluminescent panels attached back-to-back and masked with opaque black tape and neutral density filter material so it would appear to be a window frame of some thickness, with shadows on the mullions simulating illumination from above. The smaller and physically closer end of the trapezoidal window, $21.0 \mathrm{~cm}$ in height, was located $100.0 \mathrm{~cm}$ from the observer's eyes when the head was at the center of its lateral motion path. The larger end, $28.5 \mathrm{~cm}$ in height, was the more distant end, with a $26.9-\mathrm{cm}$ separation between the two ends. The luminance of the unmasked area of the window was $1.9 \log$ units above foveal threshold under the average adaptation conditions of the experiment.

To measure the observer's perceived orientation (tilt) of the trapezoidal window, a second window, a rectangular window, also with its center at eye level, was located directly in front of the observer such that when it was illuminated and the trapezoidal window was turned off, it appeared (as seen by transmission through the partially reflecting mirror) to occupy the location previously occupied by the reflected image of the trapezoidal window. Only one of the two windows was visible at any one time. The rectangular window, with cutout panes of the same number and approximate location as those of the trapezoidal window, was $26.8 \mathrm{~cm}$ high $x$ $30.5 \mathrm{~cm}$ wide and was constructed of $1 / 4$-in. plywood painted flat white. The luminance of the brightest parts of the rectangular window was $2.8 \mathrm{log}$ units above foveal threshold, and the surrounding area was covered with black cloth so as to be minimally visible. When oriented in the observer's midsagittal plane, the near edge of the rectangular window was $100.0 \mathrm{~cm}$ from the observer's eyes

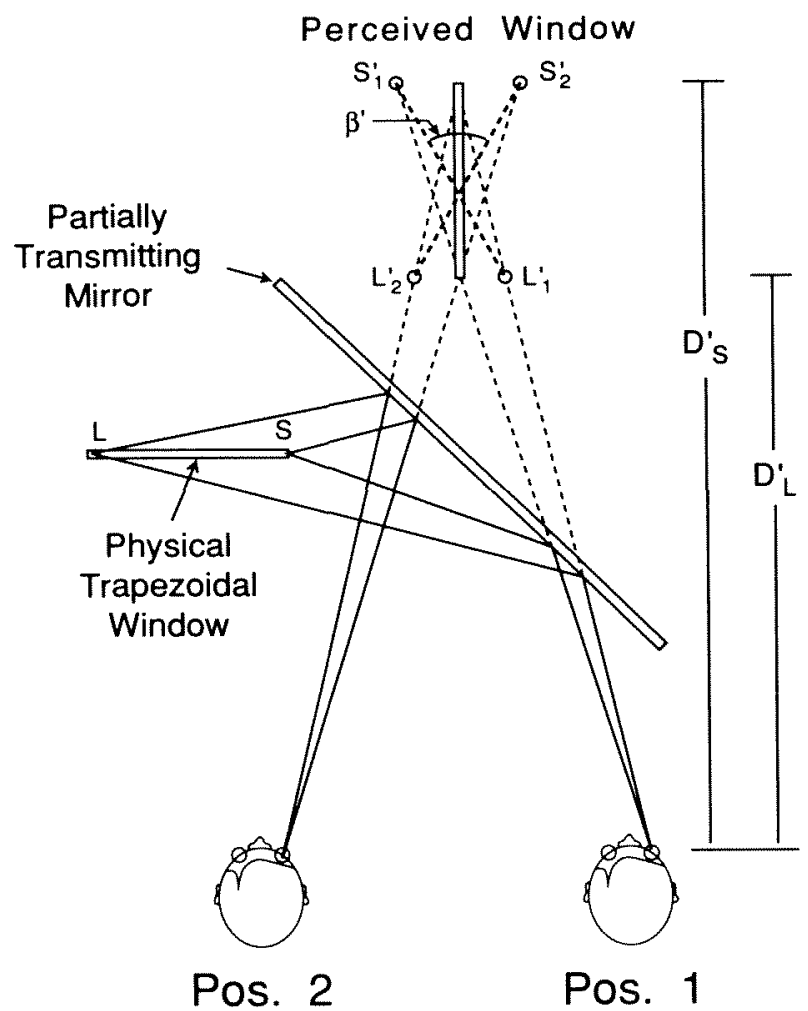

Figure 4. Top-view drawing of the method of presenting the trapezoidal window when viewed monocularly with the head either laterally moving between Positions 1 and 2 or physically stationary at those positions. Because of the illusory perspective within the trapezoidal window, its perceived depth appeared reversed at all positions of the head. This error in perceived depth resulted in the physically stationary trapezoidal window appearing to differ in orientation by an illusory angle, $\beta^{\prime}$, as measured at the extreme positions (Positions 1 and 2) of the head. 
when the head was at the midpoint of its lateral motion path. The trapezoidal window was made visible by turning on the electroluminescent panel, leaving the remainder of the visual field totally dark. The rectangular window was presented by extinguishing the trapezoidal window and turning on an overhead fluorescent lamp.

The physical orientation of the rectangular window was adjustable by the observer. For this purpose, the rectangular window was supported by a pivoting vertical shaft attached to the midpoint of its bottom edge. The shaft was connected by a series of gears to an adjustment knob located directly in front of the observer and $26.5 \mathrm{~cm}$ below eye level. To indicate the perceived orientation of the trapezoidal window, the observer turned the knob until the rectangular window appeared to have the same orientation (tilt) as the immediately previous presentation of the trapezoidal window. Unlike the monocular presentation of the trapezoidal window, the rectangular window always was observed binocularly. An IBM PC computer was used to record the tilt of the rectangular window by means of an $\mathrm{A} / \mathrm{D}$ converter connected to the potentiometer attached to the pivoting shaft supporting the rectangular window. As is indicated by the dotted lines in Figure 4, the illusory depth of the trapezoidal window is expected to change its perceived orientation, or, in the case of the dynamic condition, to appear to rotate, through an angle $\beta^{\prime}$ between $S_{1}^{\prime} L_{1}^{\prime}$ and $S_{2}^{\prime} L_{2}^{\prime}$ as the head is displaced statically or is moved dynamically between lateral Positions 1 and 2 .

\section{Procedure}

The observer was shown how to use the movable chinrest and to adjust the rectangular window. As in Experiments 1 and 2, a dynamic and a static condition were presented on separate sessions separated by approximately 1 week. In the dynamic condition, the observer continuously viewed the trapezoidal window while moving the head left and right, and in the static condition, the trapezoidal window was viewed with a stationary head only at either the right (Position 1) or the left (Position 2) extreme of the motion path of the head.

In the static condition, the observer positioned the head in the chinrest, which was immobilized at either the right or left extreme head position, and viewed the trapezoidal window monocularly with an eye patch worn over the left eye. To ensure that the expected illusory depth orientation was perceived before the experiment proceeded further, at the start of each trial, the observer was asked which side (right or left) of the trapezoidal window appeared more distant. Observation of the trapezoidal window continued until the observer signaled readiness to indicate its perceived orientation. The trapezoidal window was then turned off, the eye patch was raised for binocular vision, and the rectangular window was illuminated. The observer then adjusted the knob, which rotated the rectangular window to a position to duplicate the remembered orientation of the previously seen trapezoidal window. After the adjustment was recorded, the observer returned the rectangular window to its initial orientation at the start of the adjustment, which was midway between the two extremes of head motion or of static displacement of the head. Approximately 25 sec elapsed between trials.

The dynamic condition followed the same procedure as the static condition except that before the trapezoidal window was presented, the observer started moving the head left and right in the chinrest in time with the metronome clicks. The head motion was continued throughout the viewing of the trapezoidal window, and the observer was instructed to remember the orientation that was perceived when the chinrest was at the left or right extreme position, as specified before each trial. When the observer signaled readiness to respond, the trapezoidal window was turned off, and the head was stopped at the extreme lateral head position from which the observation was made. After the rectangular window was illuminated, the observer lifted the eye patch and turned the knob, which adjusted the rectangular window to duplicate the orientation perceived in the presentation of the trapezoidal window at the designated extreme of head motion.

The order of dynamic versus static sessions and the first side (extreme head position) from which the observations were made were counterbalanced, as they were in Experiments 1 and 2. Also, similar to Experiments 1 and 2, each session began with a practice trial that used the experimental condition presented first in that session. Two trials were run consecutively at the same head position, after which the alternative position was used.

\section{Results and Discussion}

The results obtained from the means of the two consecutive measures of the perceived orientation of the trapezoidal window averaged over the 12 observers is shown in Table 2. The perception of the orientation of the trapezoidal window is measured in degrees from the midsagittal plane located midway between the two extremes of head motion or of static head displacement. A counterclockwise deviation is positive; a clockwise deviation is negative. The differences in the perceived orientation $\left(\beta^{\prime}\right)$ between the judgments made when the head was at the extreme left and right positions also is shown in Table 2.

The differences between the perceived orientation responses were analyzed by an ANOVA on three variables: static versus dynamic head motion, head position ( 1 or 2 ) at which the response was made, and first versus second response measures. None of the factors or their interactions resulted in statistical significance at the .05 level except the head position at which the perceived orientation was measured $[F(1,11)=605.11, p<.0005]$. An ANOVA of $\beta^{\prime}$, as calculated from the response differences between the right and left extreme head positions (1 or 2 ), was completed by using the factors of static versus dynamic head motion and first versus second response measures. Neither the factors nor their interaction was statistically significant at the .05 level.

Experiment 3 clearly indicates that the errors in perceived depth considered in Experiments 1 and 2 to produce

Table 2

Perceived Orientation of the Trapezoidal Window (in Degrees) in Experiment 3

\begin{tabular}{|c|c|c|c|c|c|c|c|c|c|c|c|}
\hline \multicolumn{6}{|c|}{ Stationary (Static) Head } & \multicolumn{6}{|c|}{ Moving (Dynamic) Head } \\
\hline \multicolumn{2}{|c|}{$\begin{array}{l}\text { Head Right } \\
\text { (Position 1) }\end{array}$} & \multicolumn{2}{|c|}{$\begin{array}{c}\text { Head Left } \\
\text { (Position 2) } \\
\end{array}$} & \multicolumn{2}{|c|}{$\beta^{\prime}$ Static } & \multicolumn{2}{|c|}{$\begin{array}{l}\text { Head Right } \\
\text { (Position 1) }\end{array}$} & \multicolumn{2}{|c|}{$\begin{array}{c}\text { Head Left } \\
\text { (Position 2) } \\
\end{array}$} & \multicolumn{2}{|c|}{$\beta^{\prime}$ Dynamic } \\
\hline$M$ & $S D$ & $M$ & $S D$ & $M$ & $S D$ & $M$ & $S D$ & $M$ & $S D$ & $M$ & $S D$ \\
\hline 19.00 & 3.32 & -20.58 & 3.68 & 39.58 & 6.21 & 19.42 & 3.11 & -20.17 & 3.05 & 39.59 & 5.62 \\
\hline
\end{tabular}

Note-In Position 1, the perceptually farther end of the window $\left(S_{1}^{\prime}\right)$ always was perceived to be to the left of the perceptually nearer end of the window $\left(L_{1}^{\prime}\right)$. In Position 2 , the perceptually farther end of the window $\left(S_{2}^{\prime}\right)$ always was perceived to be to the right of the perceptually nearer end of the window $\left(L_{2}^{\prime}\right)$. (See Figure 4.) A counterclockwise deviation is positive; a clockwise deviation is negative. 
the illusory orientations and rotations of the stimulus are not limited to depth errors produced by misleading binocular disparity. Illusory perspective cues can produce similar orientation or rotation errors independently of whether the observer's head is moving or is stationary.

A finding not necessarily expected in Experiment 3 was the inability of the relative motion parallax produced by the moving head to disturb the similarity of the results obtained in the static and dynamic conditions. Unlike Experiment 2 , the trapezoidal window contains a distribution of vertical contours, and monocular viewing eliminated the binocular disparity that produced the errors in perceived depth in Experiment 1. Thus, relative motion parallax as a veridical cue of depth might have been expected to reduce the effect of the illusory perspective cue in the trapezoidal window. If this had happened, the perceived tilt of the trapezoidal window would have differed in the dynamic and static head conditions, but the difference would not have been due to any process involving anticipation. Clearly, this did not occur.

\section{GENERAL DISCUSSION}

The illusory rotation of a stimulus object viewed while moving the head laterally has been thought to be a consequence of applying compensation or reasoning-like processes to situations in which the depth within the stimulus objects is misperceived. Similarly, the accurate perception of object motion or stationariness would be explained from this point of view by the presence of appropriate amounts of compensation or inferential corrections. The experiments of the present study compare the orientation responses to the stimulus obtained in static and dynamic motion conditions and support a different explanation. It is concluded that both errors or veridicality in perceived rotation or in the perceived stationariness of stimuli extended in depth can be understood entirely in terms of the perceived distances and perceived directions of the parts of the stimulus object. If the distances of and directions to the stimulus parts are perceived accurately, the physically stationary or physically rotating object will accurately appear to be as it is physically. If the perceived distances of, or directions to, the stimulus parts are differently in error, the physically stationary or moving stimulus object will have an illusory orientation or rotation. The illusory motion will equal the perceived motion that would occur if a stimulus object were to physically move the same amount as perceived and was correctly perceived in depth and direction from the observer throughout its physical motion. This explanation is consistent with a theory of phenomenal geometry in which the basic factors of perceived distances and perceived directions from the observer determine the derived perceptions of stimulus orientation and rotation.

In addition to concluding that no processes other than those responsible for the perception of distance and direction are needed in the explanation of perceived tilt or rotation, the theory of phenomenal geometry states that it is the perceptions of distance and direction, regardless of whether these are veridical or in error, and not simply the cues of distance and direction, whether provided by actual or virtual conditions, that are important. If this conclusion is valid, it follows that perceptual learning (recalibration) or potentiation (Ebenholtz, 1976) that modifies the relationship between sensory input and the perception of orientation or rotation must achieve this modification by changing either or both the perceptions of distance and direction. If the recalibration also modifies the perception of the common motion of the stimulus, indicated by $K$ in Figures $1 \mathrm{~b}$ and $1 \mathrm{c}$, the observer's perception of self motion while viewing the stimulus also must be considered in explaining the effects of learning or potentiation.

\section{REFERENCES}

AMEs, A., JR. (1951, September). Visual perception and the rotating trapezoidal window. Psychological Monograph, 65(7, Whole No. 324).

Ebenholtz, S. M. (1976). Additivity of aftereffects of maintained head and eye rotations: An alternative to recalibrations. Perception \& Psychophysics, 19, 113-116.

Epstem, W. (1973). The process of 'taking-into-account' in visual perception. Perception, 2, 267-285.

EPSTEIN, W. (1977). What are the prospects for a higher-order stimulus theory of perception. Scandinavian Journal of Psychology, 18, 164-171.

GoGEL, W. C. (1979). The common occurrence of errors of perceived distance. Perception \& Psychophysics, 25, 2-11.

Gogel, W. C. (1980). The sensing of retinal motion. Perception \& Psychophysics, 28, 155-163.

GOGEL, W. C. (1990). A theory of phenomenal geometry and its applications. Perception \& Psychophysics, 48, 105-123.

Peterson, M. A., \& Shyi, G. C.-W. (1988). The detection of real and apparent concomitant rotation in a three-dimensional cube: Implications for perceptual interactions. Perception \& Psychophysics, 44, $31-42$.

Rock, I. (1983). The logic of perception. Cambridge, MA: MIT Press. Wallach, H. (1985, May). Perceiving a stable environment. Scientific American, pp. 118-124.

WALLACH, H. (1987). Perceiving a stable environment when one moves. Annual Review of Psychology, 38, 1-27.

Wallach, H., \& Flaherty, E. W. (1975). A compensation for field expansion caused by moving forward. Perception \& Psychophysics, 17, 445-449.

Wallach, H., Stanton, L., Becker, D. (1974). The compensation for movement-produced changes of object orientation. Perception \& Psychophysics, 15, 339-343.

(Manuscript received August 16, 1991; accepted for publication October 23, 1991.) 